\title{
Social Issues of Power Harvesting as Key Enables of WSN in Pervasive Computing
}

\author{
J. Berzosa and J. Mabe \\ Unit of Electronics and Communication \\ Tekniker-ik4 \\ Eibar 20600, Spain \\ \{jberzosa,jmabe\}@tekniker.es
}

\author{
N. J. Grabham and M.J. Tudor \\ School of Electronics and Computer Science \\ University of Southampton \\ Southampton, SO17 1BJ, UK \\ \{njg,mjt\}@ecs.soton.ac.uk
}

\begin{abstract}
Pervasive systems have gained popularity and open the door to new applications that will improve the quality of life of the users. Additionally, the implementation of such systems over an infrastructure of Wireless Sensor Networks has been proven to be very powerful. To deal with the WSN problems related to the battery of the elements or nodes that constitute the WSN, Power Harvesting techniques arise as good candidates. With $\mathrm{PH}$ each node can extract the energy from the surrounding environment. However, this energy source could not be constant, affecting the continuity and quality of the services provided. This behavior can have a negative impact on the user's perception about the system, which could be perceived as unreliable or faulty.

In the current paper, some related works regarding pervasive systems within the home environment are referenced to extrapolate the conclusions and problems to the paradigm of Power Harvesting Pervasive Systems from the user perspective. Besides, the paper speculates about the approach and methods to overcome these potential problems and presents the design trends that could be followed.
\end{abstract}

Keywords- power harvesting; pervasive; home networking; building automation; WSN

\section{INTRODUCTION}

Pervasive systems need as much information about the surrounding environment as possible in order to be really useful. The more information or interaction/sense points are available the easier it is to infer/discriminate situations or behaviours and the richer is the quality of the potential services. Therefore, it could be stated that increasing the amount of information that a system perceives brings the improvement of the cognitive capacity of the user oriented services.

It is easy to understand why WSN have gained popularity in the world of the pervasive systems. Each element of the network is relatively cheap and it can be installed nearly everywhere thanks to the wireless communication capacity and mesh routing. They can be deployed in bulk quantities of hundreds or thousands forming mesh networks with different topologies (from backbone to capillars), while each of the elements gives localized data of the environment.
Ideally pervasive systems are conceived as invisible devices embedded in the environment, providing services seamlessly to the users. However, achieving the ideal approach in the real world implementations is not totally feasible but, at least, the complexity of the system should be hidden and the interaction with the user should be provided with natural or very simple interfaces. Nevertheless, each element of a WSN has a determined life cycle due to batteries, obsolete software or other reasons. In a distributed system where each element is integrated within the environment, physical maintenance/management can be the mayor drawback. To avoid this problem, the devices must be designed to offer an autonomous operation (zero maintenance) or allow remote maintenance.

Even though remote management/maintenance is a common feature in WSNs, the problem regarding the lifetime of the batteries still remains. The first (obvious) choice would be to power devices permanently. However, besides increasing system cost (due to installation and raw material requirement), this approach clearly goes against the primary WSN nature (removing communication wires). In this situation the Power Harvesting systems constitute a sensible alternative. They provide the devices with the energy from the environment itself, increasing their life cycle virtually forever.

Ironically, power harvesting systems need some special management or deployment considerations. The amount of energy available for a device (and hence its services quality and continuity) depends on the amount and frequency of the physical effect or principle in which the power harvester relies. This could imply that the services offered by the system are not-continuous (or not-always available) depending on the presence and continuity of the physical effect to harvest from. The TIBUCON project [16] proposes a solution for constant and intensive monitoring of the building conditions based on a WSN powered with ambient energy. The technology will be deployed in real environments to fulfill two objectives: first validate the suitability of the self powered pervasive monitorization system in Buildings and, second, gather feedback data regarding the user perception of the self powered devices integrated into their everyday routine. The current paper tries to extrapolate the perception that potential users could have 
about such system and how the acceptance of those systems could be increased.

The rest of the paper is organized as follows. In section II the benefits and drawbacks of Power Harvesting systems are explained. Section III gives a description of the TIBUCON project. In Section IV, to establish the ground of this study the related researches about the user's interaction with home networking systems and the potential relation with $\mathrm{PH}$ pervasive systems are provided. In Section $\mathrm{V}$ follows a speculation about the considerations that should be taken to effectively integrate a power harvesting pervasive system into the user's routine. Finally, Section VI presents the conclusions.

\section{POWER HARVESTING}

One of the paradigms of the WSN is to have a power consumption as low as possible in order to stretch the life cycle of the devices to the maximum and hence allow the continuity of the service. In this scenario the remaining energy of a device is known beforehand and the prediction of work cycles and when the services will start to be deficient (due to battery depletion) is a relatively easy task. When the battery is discharged it can be replaced by a new one or, in some cases, replace the device itself.

Power or Energy Harvesting is the collection of ambient energy (light, vibration, thermal, etc) from the surrounding environment [7]. It was designed as an alternative to hardwired or battery powered devices as they provide an almost perpetual green energy supply. It also has other potential benefits such as reduction of system cost (installation, raw material requirement, no battery replacement, etc) and environmental impact (depleted battery processing). As there is no need for physical access to the devices for the management and replacement of the batteries, it is easier to transparently (and invisibly) integrate them into the environment because they can be literally embedded. Obviously it is still necessary for the device general management (i.e. firmware update) to be wireless.

However, in contrast to batteries, the availability of the energy or, in other words, the probability that the energy source is held, is not easily predicted. The variability in the availability of the ambient energy imposes many challenges in the design of a WSN powered solely by means of ambient energy $[14,15]$ in terms of topology stability, dynamic routing and data reliability. This makes the management of the resources in the short-term and medium-term very difficult and could lead to unexpected service lost.

The remaining energy $E_{t}$ of a Power Harvesting system at time $t$ can be modeled as follows:

$$
\mathrm{E}_{\mathrm{t}}=\mathrm{E}_{\mathrm{st}}+\mathrm{E}_{\mathrm{at}}-\mathrm{E}_{\mathrm{ct}} \text {, }
$$

where $E_{s t}$ is the stored energy, $E_{a t}$ the available ambient energy and $\mathrm{E}_{\mathrm{ct}}$ the consumed energy at time $t$. The parameter $\mathrm{E}_{\mathrm{at}}$ can be modeled as the current available energy or as a prediction of the available energy. In this last case the model tend to be more accurate but the complexity is also increased.

There are some techniques that can be used together with power harvesting systems so their benefits are exploited while increasing the reliability of the services. However all these techniques are special cases and only can be applied in certain environments or scenarios.

a) User interaction produces the necessary energy for the service. This is an ideal case because the energy will be always available when the service is needed. However, this type of techniques is more suited for immediate services (for instance light interrupters [12], revolving doors[11] or locks[10]) and it is not easily applied to services that must be active for a long period of time (although examples, such as static bicycles, do exist).

b) Hybrid systems. A hybrid system is composed of a power harvesting module and a battery. Such systems have a live cycle much longer than conventional battery powered ones. Although it can be seen as a system which simply has a bigger battery, it could define two alternative operation modes or services: mandatory services that at always active and optional services with a variable availability that will depend on external factors.

c) Permanently Powered routers. Some of the elements of the communication network could be permanently wall powered. Elements will take the load of the network maintaining the backbone of the network architecture and routing the communication traffic of the surroundings nodes forming a star topology. This would decrease the communication load of the simpler devices with limited energy (with power harvester or not) making the most of the remaining energy. This technique is very popular in home automation (for instance with Zigbee) .

Supporting service. In this case there exists a primary system that assures the availability of the service. The WSN is a supporting system that will replace the primary system in its tasks or duties wherever possible (when enough energy available). This will help in saving energy power and increasing the life cycle of the primary system.

\section{TIBUCON PROJECT}

The idea of the TIBUCON project [16] focuses on the concept that a better knowledge of the building current conditions will lead to clever decision making and control strategies. However, this data gathering could be expensive due to installation and maintenance costs of sensors. TIBUCON proposes a solution for constant and intensive monitoring of the building conditions based on the Self Powered Multi Magnitude Wireless Sensor Networks.

The project focuses on the space heating and cooling aspects, proposing a solution beyond the existing wireless based HVAC control systems, derived from the use of Self Powered Multi Magnitude Wireless Sensor Network (SPMM-WSN) for building thermal condition monitoring.

The SP-MM-WSN completely avoids the use of cables and removable batteries, thanks to the combination of extremely energy efficient wireless communication technology, ultra low power electronics, and the power harvesting concept. The proposed multi magnitude sensor platform will allow measuring of all relevant information with a single device; and the parallel energy harvesting will allow the correct sensor node performance even if one of the 
energy sources is temporarily not available (e.g. light). The use of SP-MM-WSN therefore results in an easy-to-deploy and maintenance-free building monitoring system that makes it the ideal candidate for either new or existing HVAC installations.

One of the main part of the TIBUCON project is the deployment of the technology in real environments with a twofold objective. The first aim of the demonstration is the validation of the suitability of the self powered pervasive monitorization for the seamless energy efficiency improvement of HVAC systems in Buildings. In addition, this deployment of technology in real spaces (apartments and office premises) will give a very important feedback regarding the user perception of the self powered devices integrated in their everyday routine.

At this point, an important difference should be highlighted. The deployment in offices in contrast to the deployment in private apartments. It should be noted that the wireless solution proposed by TIBUCON is based on mesh networks, where every node rely in other similar nodes to spread their information to the central point. The deployment of this federated network in spaces with a known owner or landlord (such as office buildings or public buildings) is not a big issue since, once the solution has been accepted, the Deployment Engineer is relatively free to select the best location for the nodes, in terms of RF connectivity, present ambient energy and suitability for magnitudes to be measured.

On the other hand, the deployment in apartment buildings presents a very different scenario, where every single room of the space to be monitorized is owned by a different person. This situation could be understood as the deployment of a federated network over an unfederated space. Every single person must be convinced about the node, the technical solution, the maintenance routines and the necessity of a certain conditions (again, regarding RF, ambient energy and Magnitude to be measured) for a successful deployment. This scenario imposes many challenges in order to effectively integrate the system into the everyday lives of the inhabitants.

\section{WSNS IN BUILDINGS}

In the last years WSN in Building automation has gained more and more popularity with a special focus in energy savings $[8,9]$. Following this trend, home networks presence has also increased opening a new door to new applications and services that will improve the quality of life of the users. These two scenarios are closely related from an infrastructure point of view. It is likely (although not compulsory) that the home network becomes part of the building automation system, and that they will be extended with WSNs increasing the application range and sharing the communication infrastructure with sensors and actuators. In any case, in order to analyze the reactions and behaviors of users towards a technology they live with, home networking is more relevant than building automation systems since this last one usually have external managers and operators devoted to the system maintenance.
As stated in [2] the home network must be integrated into the domestic routine as naturally as possible in order to gain the acceptance of the inhabitants. This does not necessarily mean that it is management-free but that the task involved with the management can be introduced in the everyday chores. In the paper, two concerns are highlighted for the easy deployment and maintenance of home networks in households (without the presence of dedicated professional administrators/installers):

1) Setting Up: The setting up of the home network must take into account the routine of household members. First the positioning of the technology is aligned with the activities it performs and with the routines they are related to. Secondly, the work to undertake when installing technology must be planned so it will not disrupt the routine of the household.

2) Ongoing housekeeping: There are two tasks in which housekeeping is organized. Necessary and recurrent housekeeping tasks include the everyday chores necessary to maintain the network services working. On the other hand, occasional housekeeping tasks involve the activities that must be performed to deal with punctual problems.

In [6] the seven challenges a successful smart home must undertake are described. They take into account technical and social concerns and are summarized as follows:

1) The "Accidentally" Smart Home. While new homes may be built as "smart" from scratch, existing homes need to be retroffited to increase their smartness. How will the pervasive system be integrated in the home and how will the inhabitants adapt to it?

2) Impromptu Interoperability. Given the disparity in the nature of the devices in a home (present and future), how will they interoperate?

3) No Systems Administrator. There are no expert administrators in the home; how will the average user cope with the complexity of the system?

4) Designing for Domestic Use. Technologies are usually designed for workplaces how will they fit into a home environment routine without been disruptive?

5) Social Implications of Aware Home Technologies. How will technology at home change the social behaviors of the inhabitants?

6) Reliability. How will the reliability of the interconnected devices of a smart home be assured?

7) Inference in the Presence of Ambiguity. How much inference is required for pervasive systems to be successful?

Later, the same authors research the barriers that apparently show home networking as something complex and difficult to use because communication networks were designed to be used in research labs and companies [1]. The assumptions made for these original working environments are not necessarily applicable inside an average home:

- Professional administrator: In a home there is not expected to be an advanced trained administrator. 
- Heterogeneity: Home networks have higher heterogeneity as there is no control on the type of devices a user can install. The home network infrastructure must be able to support a wide range of applications and services and new applications may be installed into a priory unknown infrastructures.

- Privacy: The privacy expected in the home and in the workplace is simply different. In the workplace there may be no personal privacy at all whereas at home it is a major concern.

As it can be seen home networking implies a series of concerns and/or responsibilities to the inhabitants. All these problems are related to the management and maintenance of the network and need to be integrated as naturally as possible in the home so it becomes part of the everyday routine. It is expected that this same principle can be applied to pervasive systems installed in homes. It does not matter if the home network is a part of the pervasive system or not, the user should see it as "my home system" and not as something separate. Therefore, since the above problems regarding the home networking will be shared by the pervasive system, this could cause even a disruption in the pervasivity itself.

Clearly these considerations are not new to the pervasive computing area. In [13] a mathematical model to predict user acceptance over time of pervasive computing applications is described. Although it is a first approach and the model is not validated, what is interesting about this paper is that it proposes to extend technology acceptance models to include trust and integration as additional input parameters. In this context, trust refers to privacy (management of personal data) and to the system behaving as the user expects. On the other hand, integration refers to the system not interfering with the users' daily activities. These conclusions are directly aligned with the researches in user acceptance of home networking technologies shown above.

\section{CONSIDERATIONS: POWER HARVESTING AND USER} AWARENESS

The success of a system based in a Wireless Sensor Network powered with energy harvesting relies in the need of the user awareness about the nature of the services provided. These services are auxiliary or complementary but they could not be always available or infallible (if no further care is taken).

This approach contradicts the idea that pervasive computing must be invisible. The user must coexist with a system which functionality is optional or auxiliary rather than primary. The user must be aware of the fact that the devices are not infallible or totally trustworthy. In some cases, they will even need some basic care in order to work properly. In any case, it opens a new concept of devices which will offer supporting/auxiliary/optional services rather than essential/primary services.

The idea is the same as in the renewable energies applied to households. While the renewable energy is available it will be the source of electric consumption, when it is not, the standard electrical installation will be used instead. The idea is slightly different because the service (energy provision) is constant but it is clear that the renewable energy is used as an auxiliary system.

People will need to change some preconceived ideas about technology and the perception that it must be infallible (which it is not, in any case). Additionally, it does exist the idea of technology that needs care. A clear example is a mobile phone, a battery powered device. The battery needs to be recharged periodically and this period will depend in the frequency of use. In [4] the term Human-Battery Interaction is introduced as the reciprocal process which mobile phone users deal with limited battery lifetime. In the related experiments the tasks the users must perform to look after their devices' battery and increase its lifetime are clearly part of the routine. In [3] the study is complemented to identify when, how, for how long and how frequently users recharge their devices' batteries, in order to assess the extent to which energy savings can be achieved. Again the experiments show the integration of the battery recharging task in the users' everyday lives.

Although pervasive systems seek the integration in the environment as to be invisible, this does not mean literally "invisible". The papers referenced in the previous chapter show that, in order to gain user's acceptance, a pervasive system only needs to be effectively integrated into everyday routine but it does not need to be necessarily "invisible". This statement seems to be the correct approach when dealing with the integration of $\mathrm{PH}$ systems (as base/backbone of a pervasive system) into households so the potential lack of services does not disrupt the user activities. That is why it is necessary to bear some considerations in mind so the user is not frustrated and could fully exploit the resources of the system. The user must be provided with mechanism or tools to allow the integration of the services offered by the $\mathrm{PH}$ system. As an example, the following list depicts the possible mechanisms such system could provide:

- The user must know at any moment if the system is working or not and its operational level. This functionality is compulsory. If there is not a method to know the current performance of the system the user will not be able to act accordingly and make any management or planning ahead.

- The system must notify about its future availability. This allows future planning of tasks by the user and to make arrangements if the system is not going to be active.

- The user should be able to choose minimum or top priority functionalities. The user will be able to adapt the working level depending on the current or future necessities or expectations. Complex unnecessary functionalities can be turned off to allow the availability of a minimum service for a longer period of time. Obviously the system will need to notify the services available and their impact in the system's continuity on the long-term.

A good analogy could be the tasks involved in taking care of a plant at home. The user must perceive the system in the same way he or she perceives the tasks needed to maintain a plant alive. When a plant is in a suitable 
environment it has enough nutrients and water to be selfsufficient. For a $\mathrm{PH}$ system this would be when, in the formula (1), $E_{\mathrm{at}} \geq \mathrm{E}_{\mathrm{ct}}$. When the conditions are not optimal, the plant will look withered because it needs more resources than it has. If the situation gets worse, the plant may die after a few days unless there is a change in the environment and the resources availability increases. Table I shows an example of this analogy and the relation with PH systems.

TABlE I. Plants - Power Harvesting Analogy

\begin{tabular}{|c|c|c|c|}
\hline $\begin{array}{c}\text { Environment } \\
\text { or Situation }\end{array}$ & Plant & Power Harvest & Risk \\
\hline Optimal & $\begin{array}{c}\text { Plenty } \\
\text { nutrients and } \\
\text { water }\end{array}$ & $E_{a t} \geq E_{c t}$ & Very low \\
\hline Sub-optimal & $\begin{array}{c}\text { Water and } \\
\text { nutrients at } \\
\text { 50\% }\end{array}$ & $\begin{array}{c}E_{a t}=2 x E_{c t} \\
E_{a t}<E_{c t}\end{array}$ & Medium \\
\hline Aggressive & $\begin{array}{c}\text { Nowater, no } \\
\text { nutrients }\end{array}$ & $\begin{array}{c}E_{a t}=0 \\
E_{a t}<E_{c t}\end{array}$ & Very high \\
\hline
\end{tabular}

\section{CONCLUSIONS AND FUTURE WORK}

Wireless sensor networks are a powerful tool for the implementation of pervasive systems. In order to improve their integration in the environment power harvesting techniques can be used as an alternative to traditional batteries. This choice could eliminate the necessity for physical maintenance due to power requirements. However, power harvesting techniques add new challenges to the design of the system to deal with the reliability and the availability of the services.

When applied to in-home pervasive systems, the use of $\mathrm{PH}$ brings further constraints on board. Home networks already lack the necessary usability (due to its default design for advanced users) and its management is seen as complex by the average user. A set of services which availability is not continuous and that need special care in order to work properly, can frustrate the user and make it doubt the reliability of the system.

However, its integration and acceptance in the everyday life is not impossible. A clear example is the mobile phone that, despite being powered by batteries and need periodic maintenance (recharging), have been effectively introduced in the user's routines. Its maintenance is seen as something natural (although annoying).

It is clear that for the acceptance of systems which services need special periodic care in order to work properly, they must be carefully designed. The user must have a proper feedback to be aware of the functionalities the system can provide and for how long so he plans ahead the actions needed to maintain the services and integrate them in the everyday life routine.

The deployments made within the TIBUCON project will give useful feedback of the user experience. The data gathered will be used for future research in order to refine the speculations made in this paper.

\section{ACKNOWLEDGMENT}

The research leading to these results has received funding from the European Union Seventh Framework Programme (FP7/2007-2013) under grant agreement $n^{\circ}$ [260034], project named TIBUCON: Self Powered Wireless Networks for HVAC system Energy Improvements, which is part of the ICT for Sustainable growth [5] Research Program.

\section{REFERENCES}

[1] W. Keith Edwards, Rebecca E. Grinter, Ratul Mahajan, and David Wetherall. 2011. Advancing the state of home networking. Commun. ACM 54, 6 (June 2011), 62-71. DOI=10.1145/1953122.1953143.

[2] P. Tolmie, A. Crabtree, T. Rodden, C. Greenhalgh, and S. Benford, "Making the Home Network at Home: Digital Housekeeping", in Proc. ECSCW, 2007, pp.331-350.

[3] Denzil Ferreira, Anind K. Dey, and Vassilis Kostakos. 2011. Understanding human-smartphone concerns: a study of battery life. In Proceedings of the 9th international conference on Pervasive computing (Pervasive'11), Kent Lyons, Jeffrey Hightower, and Elaine M. Huang (Eds.). Springer-Verlag, Berlin, Heidelberg, 19-33.

[4] Ahmad Rahmati, Angela Qian, and Lin Zhong. 2007. Understanding human-battery interaction on mobile phones. In Proceedings of the 9th international conference on Human computer interaction with mobile devices and services (MobileHCI '07). ACM, New York, NY, USA, 265-272. DOI=10.1145/1377999.1378017.

[5] http://cordis.europa.eu/fp7/ict/sustainable-growth/.

[6] W. Keith Edwards and Rebecca E. Grinter. 2001. At Home with Ubiquitous Computing: Seven Challenges. In Proceedings of the 3rd international conference on Ubiquitous Computing (UbiComp '01), Gregory D. Abowd, Barry Brumitt, and Steven A. Shafer (Eds.). Springer-Verlag, London, UK, UK, 256-272.

[7] Weddell, A. S., Harris, N. R. and White, N. M. (2008) Alternative Energy Sources for Sensor Nodes: Rationalized Design for LongTerm Deployment. In: International Instrumentation and Measurement Technology Conference, May 12-15, 2008, Victoria, British Columbia, Canada. pp. 1370-1375.

[8] Spyridon Tompros, Nikolaos Mouratidis, Michael Caragiozidis, Halid Hrasnica, and Anastasius Gavras. 2008. A pervasive network architecture featuring intelligent energy management of households. In Proceedings of the $1 \mathrm{st}$ international conference on PErvasive Technologies Related to Assistive Environments (PETRA '08). ACM, New York, NY, USA, , Article 75. DOI=10.1145/1389586.1389673.

[9] Hynek Raisigel, Gilles Chabanis, Isabelle Ressejac, Michel Trouillon, "Autonomous Wireless Sensor Node for Building Climate Conditioning Application," Sensor Technologies and Applications, International Conference on, pp. 68-73, 2010 Fourth International Conference on Sensor Technologies and Applications, 2010.

[10] GreenACCESS- energy harvesting locks for access and building control.

[11] http://www.fluxxlab.com/projects/.

[12] http://www.enocean.com/en/enocean_modules/PTM200.

[13] Connelly, K. "On developing a technology acceptance model for pervasive computing," 9th International Conference on Ubiquitous Computing (UBICOMP)-Workshop of Ubiquitous System Evaluation (USE), Springer, Innsbruck, Austria, 2007, p. 520.

[14] Seah, W. K. G., Eu, Z. A., \& Tan, H.-P. (2009). Wireless sensor networks powered by ambient energy harvesting (WSN-HEAP) Survey and challenges. 2009 1st International Conference on Wireless Communication Vehicular Technology Information Theory and Aerospace Electronic Systems Technology, 1-5.

[15] Hakan Erkal, F. Mehmet Ozcelik, Mehmet Akif Antepli, B. T. Bacinoglu, Elif Uysal-Biyikoglu: A Survey of Recent Work on Energy Harvesting Networks. ISCIS 2011: 143-147.

[16] http://www.tibucon.eu/. 